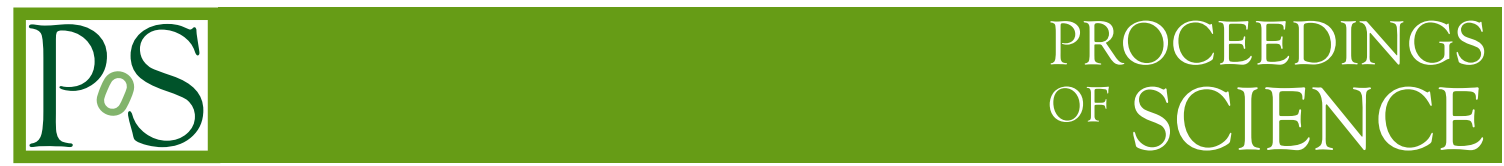

\title{
One-loop amplitudes with off-shell gluons
}

\author{
A. van Hameren ${ }^{* \dagger}$ \\ Institute of Nuclear Physics Polish Academy of Sciences, PL-31342 Kraków, Poland \\ E-mail: hameren@ifj.edu.pl
}

I report on recent progress in the calculation of cross sections within factorization prescriptions that require initial-state partons with non-vanishing transverse momentum, in particular the advancement towards one-loop amplitudes.

13th International Symposium on Radiative Corrections

24-29 September, 2017

St. Gilgen, Austria

\footnotetext{
* Speaker.

${ }^{\dagger}$ This work was supported by grant No. 2015/17/B/ST2/01838 of the National Science Center, Poland.
} 


\section{Introduction}

Factorization prescriptions involving an explicit dependence on the transverse momentum $\left(k_{T}\right)$ of one or both initial-state partons in general require partonic hard matrix elements with space-like initial-state partons. So-called $k_{T}$-factorization [1] and high-energy factorization [2,3] are wellknown examples, and a more recent one is improved transverse momentum dependent factorization [4]. Some special care must be taken in the definition and calculation of the partonic matrix elements in order to ensure gauge invariance. Several approaches exist that lead to equivalent results at tree-level [5-11]. They even allow for so-called "on-shell recursion" for amplitudes, leading to compact expressions remarkably similar to those for fully on-shell amplitudes $[12,13]$. Tree-level calculations have been completely automated in the parton-level Monte Carlo program KATIE [14]. It can be used in combination with TMDlib [15] to produce fully exclusive parton-level event files for arbitrary processes within the Standard Model.

In order to reach higher precision, calculations need to be performed at next-to-leading order (NLO). The advancement to NLO is a non-trivial step that is also necessary to assess the reliability of the factorization procedure. In order to be physically relevant, it must allow for a systematic treatment of the divergences that accompany higher-order calculations within quantum chromodynamics. Within the so-called parton reggeization approach some results have already been obtained [16-20]. Complications arise in the calculation of one-loop amplitudes, necessary for the virtual contribution in NLO calculations, due to the occurrence of linear denominators if space-like initial-state partons are involved. The resulting divergencies cannot be straightforwardly regularized with dimensional regularization. A regularization was proposed recently in [21] that comes naturally with the approach to define off-shell amplitudes from [8]. Lorentz covariance and gauge invariance are respected manifestly, and well-known techniques for the calculation of one-loop amplitudes are applicable to large degree.

\section{KaTie (https://bitbucket.org/hameren/katie/downloads/)}

KATIE is a parton-level event generator designed in a philosophy similar to HELAC [22] and ALPGEN [23], but with the extra feature that it allows for an explicit non-vanishing transverse momentum for the initial-state partons. Its output comes in the form of event files in the LHEF format [24], or in a custom file format, for the latter of which KATIE also provides the necessary histogram routines to plot arbitrary distributions. For collinear PDFs and for the running coupling KATIE relies on LHAPDF [25], while transverse momentum dependent PDFs (TMDPDFs) can be provided by TMDlib [15], or as ASCII files containing hyper-rectangular grids which KATIE interpolates itself. A hadron-level process is completely defined in a single input file. Consider for example the process $p p \rightarrow b \bar{b} \mu^{+} \mu^{-}$, for which the input file must contain

- the list of desired partonic subprocesses, e.g.

$$
g^{*} g^{*} \rightarrow b \bar{b} \mu^{+} \mu^{-} \quad, \quad u^{*} \bar{u}^{*} \rightarrow b \bar{b} \mu^{+} \mu^{-} \quad, \quad d^{*} \bar{d}^{*} \rightarrow b \bar{b} \mu^{+} \mu^{-}
$$

- the identifier for the collinear PDF set in LHAPDF;

- the identifier for the TMDPDF set in TMDlib, or alternatively a list of grid files and the directory where they can be found; 
- the value of the center-of-mass energy and a list of phase space cuts;

- the desired values of couplings and masses and widths of the particles involved;

- the desired interaction, e.g. both QCD and electroweak interactions for this example.

For each partonic sub-process, the phase space sampler is optimized seperately. This stage is rather quick, compared to the second stage during which the actual events are generated. This process can trivially be parallelized by running instances of an executable with different seeds for the random number generator. A few recent studies in which KATIE has been employed can be found in [26-29].

\section{One-loop amplitudes}

In [8] amplitudes with off-shell gluons were defined at tree-level by introducing auxiliary quark-antiquark pairs and embedding the process into an on-shell process involving those instead of the off-shell gluons. Let $p^{\mu}$ be the momentum of one of the scattering hadrons the off-shell gluon is imagined to originate from. The momentum of latter has explicit transversal components and is given by $k^{\mu}=x p^{\mu}+k_{T}^{\mu}$. The momenta $p_{A}^{\mu}, p_{A^{\prime}}^{\mu}$ of the auxiliary quark-antiquark are chosen such that they add up to the desired momentum $k^{\mu}$ for the off-shell gluon. This can for example be achieved as follows:

$$
p_{A}^{\mu}=\Lambda p^{\mu}+\alpha q^{\mu}+\beta k_{T}^{\mu} \quad, \quad p_{A^{\prime}}^{\mu}=x p^{\mu}+k_{T}^{\mu}-p_{A}^{\mu},
$$

where $q^{\mu}$ is an arbitrary light-like momentum with $p \cdot q>0$, where $q \cdot k_{T}=p \cdot k_{T}=0$, and where

$$
\alpha=\frac{-\beta^{2} k_{T}^{2}}{\Lambda(p+q)^{2}} \quad \text { with } \quad \beta=\frac{1}{1+\sqrt{1-x / \Lambda}} .
$$

The momenta $p_{A}, p_{A^{\prime}}$ are then guaranteed to satisfy the desired relations

$$
p_{A}^{2}=p_{A^{\prime}}^{2}=0 \quad, \quad p_{A}^{\mu}+p_{A^{\prime}}^{\mu}=x p^{\mu}+k_{T}^{\mu}
$$

for any value of the parameter $\Lambda$. The amplitude with the off-shell gluon is obtained after taking the limit of large $\Lambda$ :

$$
\frac{\left|k_{T}\right|}{\Lambda} \mathscr{A}\left(\emptyset \rightarrow \overline{\mathrm{q}}_{A}\left(p_{A}(\Lambda)\right) \mathrm{q}_{A}\left(p_{A^{\prime}}(\Lambda)\right)+X\right) \stackrel{\Lambda \rightarrow \infty}{\longrightarrow} \mathscr{A}\left(\emptyset \rightarrow g^{*}\left(x p+k_{T}\right)+X\right) .
$$

The symbol $X$ stands for other particles in the partonic process, for example $g b \bar{b} \mu^{+} \mu^{-}$in the process $g^{*} g \rightarrow b \bar{b} \mu^{+} \mu^{-}$.

The limit of large $\Lambda$ is rather straightforward for tree-level amplitudes, and boils down to the limit

$$
\mathrm{i} \frac{\not p_{A}+\not K}{\left(p_{A}+K\right)^{2}} \stackrel{\Lambda \rightarrow \infty}{\longrightarrow} \frac{\mathrm{i} \not p}{2 p \cdot K}
$$

for the auxiliary quark propagators. On the integrand of a one-loop amplitude with an auxiliary quark-antiquark pair, the limit leads to linear denominators of the type $2 p \cdot K$ where $K^{\mu}$ contains the 
loop integration momentum $\ell^{\mu}$. The divergencies caused by such denominators cannot straightforwardly be regulated with dimensional regularization. Recently, [21] proposed to use $\Lambda$ as the regulator, and perform (3.5) after the loop integration. Since all loop denominators are quadratic before the limit, the loop integrals are well-defined within dimensional regularization. The divergencies occur as powers of $\log \Lambda$.

One way to calculate off-shell amplitudes would be to take the limit of large $\Lambda$ on expressions involving the auxiliary quark-antiquark pair. This would realistically only be possible for simple processes, and one would rather like to be able to apply for example so-called integrand-based reduction methods [30,31]. Much attention is payed in [21] to complications due to the noncommutativity of counting powers of $\Lambda$ and performing the loop integral, and care has to be taken in identifying all non-vanishing contributions in the limit $\Lambda \rightarrow \infty$ when applying the mentioned reduction methods.

\section{References}

[1] L. V. Gribov, E. M. Levin and M. G. Ryskin, Phys. Rept. 100 (1983) 1. doi:10.1016/0370-1573(83)90022-4

[2] S. Catani, M. Ciafaloni and F. Hautmann, Nucl. Phys. B 366 (1991) 135. doi:10.1016/0550-3213(91)90055-3

[3] J. C. Collins and R. K. Ellis, Nucl. Phys. B 360 (1991) 3. doi:10.1016/0550-3213(91)90288-9

[4] P. Kotko, K. Kutak, C. Marquet, E. Petreska, S. Sapeta and A. van Hameren, JHEP 1509 (2015) 106 doi:10.1007/JHEP09(2015)106 [arXiv:1503.03421 [hep-ph]].

[5] L. N. Lipatov, Nucl. Phys. B 452 (1995) 369 doi:10.1016/0550-3213(95)00390-E [hep-ph/9502308].

[6] L. N. Lipatov and M. I. Vyazovsky, Nucl. Phys. B 597 (2001) 399 doi:10.1016/S0550-3213(00)00709-4 [hep-ph/0009340].

[7] E. N. Antonov, L. N. Lipatov, E. A. Kuraev and I. O. Cherednikov, Nucl. Phys. B 721 (2005) 111 doi:10.1016/j.nuclphysb.2005.05.013, 10.1016/j.nuclphysb.2005.013 [hep-ph/0411185].

[8] A. van Hameren, P. Kotko and K. Kutak, JHEP 1301 (2013) 078 doi:10.1007/JHEP01(2013)078 [arXiv:1211.0961 [hep-ph]].

[9] A. van Hameren, K. Kutak and T. Salwa, Phys. Lett. B 727 (2013) 226 doi:10.1016/j.physletb.2013.10.039 [arXiv:1308.2861 [hep-ph]].

[10] P. Kotko, JHEP 1407 (2014) 128 doi:10.1007/JHEP07(2014)128 [arXiv:1403.4824 [hep-ph]].

[11] A. Karpishkov, M. Nefedov and V. Saleev, arXiv:1707.04068 [hep-ph].

[12] A. van Hameren, JHEP 1407 (2014) 138 doi:10.1007/JHEP07(2014)138 [arXiv:1404.7818 [hep-ph]].

[13] A. van Hameren and M. Serino, JHEP 1507 (2015) 010 doi:10.1007/JHEP07(2015)010 [arXiv:1504.00315 [hep-ph]].

[14] A. van Hameren, arXiv:1611.00680 [hep-ph].

[15] F. Hautmann, H. Jung, M. Krämer, P. J. Mulders, E. R. Nocera, T. C. Rogers and A. Signori, Eur. Phys. J. C 74 (2014) 3220 doi:10.1140/epjc/s10052-014-3220-9 [arXiv:1408.3015 [hep-ph]].

[16] M. Hentschinski and A. Sabio Vera, Phys. Rev. D 85 (2012) 056006 doi:10.1103/PhysRevD.85.056006 [arXiv:1110.6741 [hep-ph]]. 
[17] G. Chachamis, M. Hentschinski, J. D. Madrigal Martínez and A. Sabio Vera, Phys. Rev. D 87 (2013) no.7, 076009 doi:10.1103/PhysRevD.87.076009 [arXiv:1212.4992 [hep-ph]].

[18] G. Chachamis, M. Hentschinski, J. D. Madrigal Martínez and A. Sabio Vera, Nucl. Phys. B 876 (2013) 453 doi:10.1016/j.nuclphysb.2013.08.013 [arXiv:1307.2591 [hep-ph]].

[19] M. Nefedov and V. Saleev, arXiv:1608.04201 [hep-ph].

[20] M. Nefedov and V. Saleev, arXiv:1709.06246 [hep-th].

[21] A. van Hameren, arXiv:1710.07609 [hep-ph].

[22] A. Cafarella, C. G. Papadopoulos and M. Worek, Comput. Phys. Commun. 180 (2009) 1941 doi:10.1016/j.cpc.2009.04.023 [arXiv:0710.2427 [hep-ph]].

[23] M. L. Mangano, M. Moretti, F. Piccinini, R. Pittau and A. D. Polosa, JHEP 0307 (2003) 001 doi:10.1088/1126-6708/2003/07/001 [hep-ph/0206293].

[24] J. Alwall et al., Comput. Phys. Commun. 176 (2007) 300 doi:10.1016/j.cpc.2006.11.010 [hep-ph/0609017].

[25] A. Buckley, J. Ferrando, S. Lloyd, K. Nordström, B. Page, M. Rüfenacht, M. Schönherr and G. Watt, Eur. Phys. J. C 75 (2015) 132 doi:10.1140/epjc/s10052-015-3318-8 [arXiv:1412.7420 [hep-ph]].

[26] R. Maciuła and A. Szczurek, Phys. Rev. D 94 (2016) no.11, 114037 doi:10.1103/PhysRevD.94.114037 [arXiv:1610.01810 [hep-ph]].

[27] R. Maciuła and A. Szczurek, Phys. Rev. D 96 (2017) no.7, 074013 doi:10.1103/PhysRevD.96.074013 [arXiv:1707.08366 [hep-ph]].

[28] M. Deák, K. Kutak and K. Tywoniuk, Eur. Phys. J. C 77 (2017) no.11, 793 doi:10.1140/epjc/s10052-017-5358-8 [arXiv:1706.08434 [hep-ph]].

[29] A. van Hameren, M. Kłusek-Gawenda and A. Szczurek, Phys. Lett. B 776 (2018) 84 doi:10.1016/j.physletb.2017.11.029 [arXiv:1708.07742 [hep-ph]].

[30] G. Ossola, C. G. Papadopoulos and R. Pittau, Nucl. Phys. B 763 (2007) 147 doi:10.1016/j.nuclphysb.2006.11.012 [hep-ph/0609007].

[31] R. K. Ellis, W. T. Giele and Z. Kunszt, JHEP 0803 (2008) 003 doi:10.1088/1126-6708/2008/03/003 [arXiv:0708.2398 [hep-ph]]. 